\title{
Una Revisión de la Salud Mental Materna en Tiempos de Covid-19
}

\author{
A Review of the Maternal Mental Health in the Time of Covid-19
}

\section{Claudia Parasi ${ }^{1}$}

\section{RESUMEN}

En el presente artículo se realiza una revisión de la evidencia más reciente sobre la salud mental materna en tiempos de Covid-19. Se revisan seis artículos provenientes de diferentes países que describen estudios con muestras superiores a las 200 personas, reportando la prevalencia de ansiedad y/o depresión de madres en contexto de pandemia. En todos los estudios se registra un aumento de la prevalencia de la ansiedad y/o depresión a partir de la aparición de la Covid-19. Finalmente, también se realiza una recopilación de los principales aportes propuestos en los estudios revisados para reducir las consecuencias negativas asociadas al aumento de ansiedad y depresión.

PALABRAS CLAVE: ansiedad, depresión, salud mental, madres, covid-19.

\section{SUMMARY}

This article reviews the most recent evidence on maternal mental health in times of Covid-19. Six articles from different countries are reviewed, describing studies with samples of more than 200 people and reporting prevalence of anxiety and/or depression among mothers in the context of the current pandemic. In all the studies, an increase in the prevalence of anxiety and/or depression was recorded after the appearance of Covid-19. Finally, it is made a compilation of the main contributions proposed in the reviewed articles to reduce the negative consequences associated with increased anxiety and depression.

KEYWORDS: anxiety, depression, mental health, mothers, covid-19.

\section{INTRODUCCIÓN}

Laactual pandemia ocasionada por elvirus SarsCoV-2, ha traído consigo cambios importantes en la vida de las personas a nivel mundial. Se sabe que esta enfermedad resulta particularmente peligrosa para los grupos de riesgo, tales como: adultos mayores y enfermos crónicos (Pérez et al., 2020). A pesar de que no se han reportado gran número de casos graves en gestantes y bebés y que la mayoría de gestantes son asintomáticas (Mullins et al., 2020; Chen et al., 2020; Guevara-Ríos et al., 2020), es importante no perder de vista lo que viene sucediendo en la salud mental materna en un contexto tan atípico como el que vivimos en la actualidad, caracterizado por aislamiento social, temor al contagio e inestabilidad y por la dificultad en los servicios sanitarios para abordar a las madres y bebés de manera integral. En este contexto, muchas veces se han postergado

Práctica privada. Lima, Perú. 
las necesidades psicológicas de las madres para priorizar ciertas prácticas destinadas a prevenir y atender la infección por Covid-19, las mismas que no siempre cuentan con un respaldo en la evidencia científica (Semaan et al., 2020). Es importante comprender que, al tratarse de una nueva enfermedad, el conocimiento que se tiene sobre la misma se encuentra aún en construcción. Por otro lado, tampoco se puede ignorar la evidencia científica que se encuentra disponible acerca de los beneficios físicos y psicológicos del vínculo temprano entre la madre y el bebé, el cual se ve reflejado en conductas como: la lactancia temprana, contacto piel con piel y separación cero (Organización Panamericana de la Salud, 2018; Moore et al., 2016). Precisamente a raíz del escenario actual, se considera oportuno realizar una búsqueda de las evidencias disponibles para tener una aproximación al estado de la salud mental materna en tiempos de Covid-19.

El objetivo del presente estudio fue realizar una revisión de la evidencia científica disponible sobre el estado de la salud mental materna en tiempos de Covid-19, con la finalidad de conocer las repercusiones que la pandemia tiene sobre la esfera psicológica de la diada madre-bebé.

\section{Marco Teórico}

La salud mental materna es un tópico dentro de la salud mental que ha venido cobrando fuerza con estudios de diferentes científicos alrededor del mundo. Al hablar de salud mental materna, se debe considerar que, durante la etapa perinatal, es decir, durante el embarazo, parto y puerperio, existen diferentes factores de riesgo que podrían hacer a una mujer más proclive a desarrollar una psicopatología (Sociedad Marcé Española, 2020). En ese sentido, es importante tener en cuenta que durante el periodo perinatal, la vulnerabilidad se extiende también al recién nacido, debido a que la dimensión psicológica de la diada madre-bebé funciona de manera conjunta con la finalidad de facilitar la supervivencia de la especie (Olza, 2019). La salud mental materna está unida a la salud mental del bebé, hay una adherencia indivisible de la esfera emocional de ambos. Es importante saber que la etapa perinatal puede verse alterada por condiciones ambientales, por lo que resulta particularmente importante considerar los siguientes aspectos: promover la atención de las necesidades psicológicas de la mujer, evitar intervenciones innecesarias al momento del parto, procurar un ambiente seguro y cálido y facilitar el establecimiento del vínculo temprano de la díada a través del contacto piel con piel, la lactancia temprana y la nula separación de madre y bebé (Moore et al., 2016). Lamentablemente, a raíz de la pandemia se han propuesto ciertas prácticas en los centros de salud que muchas veces ocasionan la separación de los miembros de la díada, así como el aumento del riesgo de sufrir violencia obstétrica (Sadler et al., 2020). A propósito de esto, la OMS ha publicado una guía para el abordaje de pacientes con Covid-19, donde señala la importancia de mantener la lactancia materna y evitar la separación entre madre y bebé, lo que ha generado que en algunos países se modifiquen sus protocolos de actuación al respecto (OMS, 2020). Estudios realizados en Inglaterra y Noruega, que datan de los primeros meses del año 2020, ya señalaban las posibles consecuencias negativas de la pandemia en la salud mental materna, manifestadas principalmente en problemas psicosociales (Topalidou et al., 2020; Thapa et al., 2020). Eventualmente, el tema de la salud mental perinatal ya preocupaba desde el inicio de la pandemia, debido a la vulnerabilidad de este grupo en particular por el período de vida que atraviesa. En el Perú aún no existen estudios minuciosos sobre la salud mental perinatal en contexto de la pandemia. Por tal motivo se procede a realizar una revisión de la evidencia internacional al respecto.

\section{MATERIAL Y METODOS}

Para esta revisión, se realizó la búsqueda en las bases de datos Researchgate, Google Scholar, Dialnet y Scielo, usando las siguientes combinaciones de palabras: "mental health perinatal covid 19", "maternal mental health covid 19”. Se realizó la búsqueda de artículos en inglés y en español y se consideró solamente a los estudios que hubieran incluido una muestra de por lo menos 
200 personas y que fueran de tipo cuantitativo. Es importante señalar que actualmente existe aún escasa evidencia específica sobre el tema, sobre todo en español, ya que la mayoría de estudios que se hallaron en este idioma hacían referencia al aspecto biomédico, casi ninguno se centraba en los aspectos psicológicos asociados a la salud mental materna durante la pandemia. Por otro lado, para la búsqueda bibliográfica se consideró a las mujeres en cualquier etapa de maternidad. Finalmente, quedaron seleccionados seis artículos de diferentes países en idioma inglés para esta revisión. Las búsquedas se realizaron entre julio y setiembre del 2020.

\section{RESULTADOS}

\section{Características generales}

De los seis estudios revisados, tres provenían de Canadá, uno de Grecia, uno de Inglaterra y uno de China, respectivamente (Tabla 1). Todos estaban escritos en inglés y las muestras fueron superiores a 200 personas.

Los constructos que se eligieron para evaluar la salud mental perinatal en los estudios revisados fueron: ansiedad, en un artículo (Preis et al., 2020); depresión, en un artículo (Wu et al., 2020) y ansiedad con depresión en cuatro artículos (Cameron et al., 2020; Dagklis et al., 2020; Davenport et al., 2020; Lebel et al., 2020). La mayoría de los estudios revisados fueron realizados con mujeres embarazadas, solamente uno fue realizado con madres de niños pequeños (Cameron et al., 2020). Todos los estudios coincidieron en el aumento de síntomas asociados a malestar emocional desde que la Covid-19 se expandió.

\section{Resultados por estudios}

En Canadá se halló que hubo un aumento de 25\% de la depresión durante la pandemia (Davenport et al., 2020). En el segundo estudio realizado en Canadá se evaluó a embarazadas durante la pandemia y hallaron que el $37 \%$ presentaba depresión y el 57\% síntomas clínicos de ansiedad. Los investigadores también concluyen que los resultados obtenidos son altos en comparación con sus registros previos a la pandemia (Lebel et al., 2020). Por último, Cameron et al. (2020) también hallaron en Canadá una prevalencia del $33,16 \%$ de depresión en madres de niños de 0 a 3 años a la vez que una prevalencia de ansiedad de 36,27\%. También reportaron niveles más elevados de ansiedad y depresión en comparación con la situación pre-pandémica (Cameron et al., 2020).

En Grecia se evaluó a las participantes durante los primeros días de aislamiento y se hallaron niveles más altos de ansiedad estado (40,9\% en el nivel de ansiedad leve, $27,5 \%$ en ansiedad moderada y 9,7\% en ansiedad alta), a diferencia de la ansiedad rasgo (34,6\% en el nivel de ansiedad leve, 10,8\% en ansiedad moderada y $1,9 \%$ en ansiedad alta). Asimismo, hallaron que el $14,9 \%$ de la muestra evidenció depresión durante la primera fase de aislamiento. También encontraron un aumento de la prevalencia de la ansiedad, que inicialmente era de $15,2 \%$, mientras que la depresión no evidenció aumento significativo, ya que antes de la pandemia la prevalencia era de $16,0 \%$ (Dagklis

Tabla 1. Características generales de los estudios revisados según autores y año, país, tamaño de muestra y constructos evaluados

\begin{tabular}{llll}
\hline Autores & País & n & Constructo evaluado \\
\hline Davenport et al., 2020 & Canadá & 900 & Ansiedad y depresión \\
Dagklis et al., 2020 & Grecia & 269 & Ansiedad y depresión \\
Preis et al., 2020 & Inglaterra & 788 & Ansiedad \\
Lebel et al., 2020 & Canadá & 1987 & Ansiedad y depresión \\
Cameron et al., 2020 & Canadá & 641 & Ansiedad y depresión \\
Wu et al., 2020 & China & 4124 & Depresión \\
\hline
\end{tabular}


Tabla 2. Propuestas de mejora para la salud mental de las madres planteadas en los estudios revisados

\begin{tabular}{ll}
\hline Autores & Propuesta \\
\hline Davenport et al., 2020 & Intervención psicológica a mujeres embarazadas y en el posparto \\
Dagklis et al., 2020 & Realización de más investigaciones al respecto \\
& $\begin{array}{l}\text { Elaboración de políticas públicas, apoyo de la tele-medicina, } \\
\text { proporcionar información sobre procedimientos durante la } \\
\text { pandemia, realización de más investigaciones sobre el tema }\end{array}$ \\
Lebel et al., 2020 2020 & Fomentar mayor apoyo social y cuidado del sueño en las gestantes \\
Cameron et al., 2020 & $\begin{array}{l}\text { Elaborar estrategias de intervención temprana para hacer frente al } \\
\text { estrés, promover la comunicación efectiva } \\
\text { Wu et al., 2020 }\end{array}$ \\
& $\begin{array}{l}\text { Proveer primeros auxilios psicológicos, estrategias de } \\
\text { afrontamiento de estrés, aislamiento materno }\end{array}$ \\
\hline
\end{tabular}

et al., 2020).

En Inglaterra, los investigadores evaluaron a las participantes durante la pandemia y hallaron que el $35 ., 6 \%$ presentó niveles bajos de ansiedad, el 21,6\% nivel moderado de ansiedad y el $21,7 \%$ ansiedad severa. Concluyeron que hubo un aumento de la prevalencia durante la pandemia, comparando con sus registros antes del brote de la Covid-19 (Preis et al., 2020). Finalmente, en China se evaluó a mujeres embarazadas antes y después de haberse declarado la epidemia de la Covid-19 y hallaron un aumento en los niveles de depresión de $26 \%$ a 29.6\% (Wu et al., 2020).

\section{Propuestas de mejora}

En la Tabla 2 se presentan las principales propuestas de mejora planteadas por los autores de los estudios revisados. La mayoría coincidió en poder realizar un abordaje dirigido a las madres para dotarlas de recursos que le permitan afrontar mejor la situación de pandemia.

\section{DISCUSIÓN}

A pesar de haber pocos estudios sobre la salud mental materna en estos tiempos, se cumplió el objetivo de realizar una revisión de los hallazgos más relevantes de la evidencia disponible en la actualidad. Los resultados de la evidencia revisada demuestran un aumento de la ansiedad y depresión en mujeres embarazadas y madres de niños pequeños a partir del inicio de la pandemia por la Covid-19. Durante los primeros meses de pandemia, algunos estudios preliminares advertían sobre los riesgos y repercusiones negativas en la salud mental perinatal (Topalidou et al., 2020; Thapa et al., 2020), en la actualidad podemos afirmar que efectivamente existen consecuencias negativas derivadas de la situación de pandemia. También es importante considerar que el conocimiento al respecto se sigue construyendo, por lo que seguramente en los próximos meses se tendrá mucha más evidencia disponible con datos más contundentes, tal como el estudio internacional que viene realizando un grupo de investigadoras españolas junto a la Sociedad Marcé Española (Sociedad Marcé Española, 2020).

Por otro lado, los estudios revisados también aportaron algunas propuestas para reducir el impacto de las repercusiones psicológicas negativas derivadas de la situación de pandemia, las mismas que se recopilaron para facilitar la reflexión sobre las mismas con miras a la elaboración de estrategias que permitan a las madres atravesar de forma más llevadera su propio embarazo y puerperio en el contexto crítico que vivimos. En ese sentido, considero muy acertadas las propuestas sugeridas en los estudios revisados. En los tiempos que vivimos, es de vital importancia un acompañamiento permanente a las madres desde la etapa del embarazo, haciendo uso de herramientas tecnológicas, como las tele-consultas. Asimismo, es importante que el seguimiento sea integral, brindando espacios 
de atención psicológica, y generando redes de apoyo entre madres, con la finalidad de reducir el impacto de la soledad durante la pandemia. Sería muy importante que estas medidas se prolongaran durante el periodo puerperal e incluso durante los primeros años de vida del bebé, ya que se ha demostrado que los grupos de apoyo ayudan a prevenir el desarrollo de psicopatología en las madres (Olhaberry et al., 2013). Es importante además tener en cuenta que también durante la pandemia se presentan escenarios adversos como duelos perinatales, nacimientos de bebés prematuros, enfermedades del recién nacido, entre otras circunstancias, que pueden acrecentar la posibilidad de psicopatología materna. En ese sentido, es urgente ofrecer un acompañamiento psicológico a estas madres, aunque sea de modo virtual.

Finalmente, existe poca evidencia en español, la cual sería muy necesaria para conocer la situación en nuestros contextos latinoamericanos, y con más razón en el territorio peruano. Es por esto que resulta necesario poder continuar investigando sobre estos temas en contexto de pandemia para tener un panorama más cercano a nuestra realidad.

\section{Correspondencia:}

Claudia Parasi

Correo electrónico: psic.cparasi@gmail.com

\section{REFERENCIAS BIBLIOGRAFICAS}

Cameron, E., Joyce, K., Delaquis, C., Reynolds, K., Protudjer, J. \& Roos, L. (2020). Maternal psychological distress \& mental health service use during the COVID 19 pandemic. Journal of Affective Disorders, 276. https:// doi.org/10.31234/osf.io/a53zb

Chen, Y., Peng, H., Wang, L., Zhao, L., Zeng, L., Gao, H. \& Liu, Y. (2020). Infants born to mothers with a new coronavirus (covid-19). Frontiers in Pediatrics, 8, 2296-2360. https:// doi.org/10.3389/fped.2020.00104

Dagklis, T., Tsakiridis, I., Mamopoulus, A., Athanasiadis, A., Pearson, R. \& Papazisis, G.
(2020). Impact of the COVID-19 lockdown on antenatal mental health in Greece. Psychiatry and Clinical Neurosciences, 74, 616-617. https://doi.org/10.1111/pcn.13135

Davenport, M., Meyer, S., Meah, V., Strynadka, M. \& Khurana, R. (2020). Moms are not ok: Covid-19 and maternal mental health. Frontiers in Global Women's Health, 1. https:// doi.org/10.3389/fgwh.2020.00001

Guevara-Ríos, E., Carranza-Asmat, C. \& Zevallos-Espinoza, K. (2020). Prevalencia y caracterización de gestantes seropositivas para SARS-CoV-2. Revista Peruana de Investigación Materno Perinatal, 9(2), 13-20. https://doi.org/10.33421/inmp.2020198

Lebel, C., MacKinnon, A., Bagshawe, M., Tomfohr, L., \& Giesbrecht, G. (2020). Elevated depression and anxiety among pregnant individuals during the COVID 19 pandemic. Journal of Affective Disorders, 277, 5-13. https://doi.org/10.31234/osf.io/gdhkt

Moore, E., Bergman, N., Anderson, G. \& Medley, N. (2016). Early skin-to-skin contact for mothers and their healthy newborn infants. Cochrane Database of Systematic Reviews. https://doi.org/10.1002/14651858.CD003519. pub4

Mullins, E., Evans, D., Viner, R., O'Brien, P. \& Morris, E. (2020). Coronavirus in pregnancy and delivery: rapid review and expert consensus. MedRxiv. https://doi.org/10.1101/2 020.03.06.20032144.

Olhaberry, M., Escobar, M., San Cristóbal, P., Santelices, M., Farkas, C., Rojas, G. \& Martínez, V. (2013). Intervenciones psicológicas perinatales en depresión materna y vínculo madre-bebé: una revisión sistemática. Terapia Psicológica, 31(2), 249-261. https:// dx.doi.org/10.4067/S0718-480820130002 00011

Olza, I. (2019). El estado modificado de conciencia del parto fisiológico. Instituto Europeo de Salud Mental Perinatal. https:// saludmentalperinatal.es/2019/04/07/estadomodificado-conciencia-del-parto-fisiologico/

Organización Mundial de la Salud (2020). Clinical management of severe acute respiratory infection (SARI) when COVID-19 
disease is suspected. https://saludmental perinatal.es/wp-content/uploads/2020/03/ clinical-management-of-novel-cov-1.pdf

Organización Panamericana de la Salud. (2018). Recomendaciones de la OMS sobre atención prenatal para una experiencia positiva del embarazo. Organización Panamericana de la Salud. https://iris.paho.org/bitstream/handle/ 10665.2/49550/9789275320334_spa. pdf?ua $=1$

Perez, M. R., Gómez, J.J. \& Diéguez, R. A. (2020).Características clínico-epidemiológicas de la COVID-19. Revista Habanera de Ciencias Médicas, 19(2), e_3254. http:// www.revhabanera.sld.cu/index.php/rhab/ article/view/3254

Preis, H., Mahaffey, B., Heiselman, C. \& Lobel, M. (2020). Pandemic-related pregnancy stress and anxiety among women pregnant during the coronavirus disease 2019 pandemic. American Journal of Obstetrics and Gynecology, 2 (3). https://doi.org/10.1016/j. ajogmf.2020.100155

Sadler, M., Leiva, G. \& Olza, I. (2020). Covid-19 as a risk factor for obstetric violence. Sexual and Reproductive Health Matters, 28(1), 4648. https://doi.org/10.1080/26410397.2020.17 85379

Semaan, A., Audet, C. \& Huysmans, E. (2020). Voices from the frontline: findings from a thematic analysis of a rapid online global survey of maternal and newborn health professionals facing the COVID-19 pandemic.
BMJ Global Health, 5, e002967. https://doi. org/10.1136/bmjgh-2020-002967

Sociedad Marcé Española. (2020). El impacto de la COVID 19 sobre la maternidad: estudio internacional liderado por investigadoras españolas. Sociedad Marcé Española. http:// www.sociedadmarce.org/detall.c fm/ ID/14413/ESP/el-impacto-covid-19-sobrematernidad-estudio-internacional-lideradopor-investigadoras-espanyolas.htm

Thapa, S., Mainali, A., Schwank, S. \& Acharya, G. (2020). Maternal mental health in the time of the COVID 19 pandemic. Acta Obstetrica et Gynecologica Scandinavica, 99, 817-818. https://doi.org/10.1111/aogs.13894

Topalidou, A., Thomson, G. \& Downe, S. (2020). Covid-19 and maternal and infant health: are we getting the balance right? A rapid scoping review. The Practising Midwife, 3(7). https:// www.all4maternity.com/covid-19-andmaternal-and-infant-health-are-we-gettingthe-balance-right-a-rapid-scoping-review/

Wu, Y., Zhang, C. \& Liu, H. (2020). Perinatal depressive and anxiety symptoms of pregnant women during the coronavirus disease 2019 outbreak in China. American Journal of Obstetrics and Gynecology, 223(2). https:// doi.org/10.1016/j.ajog.2020.05.009

Recibido: 05/12/2020

Aceptado: 31/12/2020 